\title{
Three-point Green function of the stress-energy tensor in the AdS-CFT correspondence
}

\author{
G. Arutyunov* \\ Universita di Milano, Dipartimento di Matematica “Federigo Enriques" via C. Saldini, 50-20133, Milano, Italy \\ and Steklov Mathematical Institute, Gubkin str. 8 GSP-1, 117966, Moscow, Russia \\ S. Frolov ${ }^{\dagger}$ \\ Department of Physics and Astronomy, University of Alabama, Box 870324, Tuscaloosa, Alabama 35487-0324 \\ and Steklov Mathematical Institute, Gubkin str. 8, GSP-1, 117966, Moscow, Russia
}

(Received 16 February 1999; published 14 June 1999)

\begin{abstract}
We compute the three-point function of the stress-energy tensor in $d$-dimensional conformal field theory CFT from $\mathrm{AdS}_{d+1}$ gravity. For $d=4$ the coefficients of the three linearly independent conformally covariant forms entering the three-point function are exactly the same as given by the free-field computations in the $\mathcal{N}=4 \mathrm{SYM}$ theory just as expected from the known renormalization theorems. For $d=3$ and $d=6$ our results give the value of the corresponding three-point function in the theories of strongly coupled $\mathcal{N}=8$ superconformal scalar and $(2,0)$ tensor multiplets, respectively. [S0556-2821(99)01114-5]
\end{abstract}

PACS number(s): 11.25.Hf, 04.50.+h

\section{INTRODUCTION}

The Maldacena conjecture [1-3] relating the large- $N$ limit of certain conformal field theories (CFT's) in $d$ dimensions with supergravity on the product of the $(d+1)$-dimensional anti-de Sitter (AdS) space with a compact manifold has been recently tested by explicit computation of many two- and three-point gauge theory correlation functions from AdS supergravity [4-20]. Considerable progress was also achieved in studying four-point correlators [21-27], whose complete computation, however, requires the knowledge of the supergravity action on the AdS background beyond the quadratic [28] approximation.

An important question being yet unsolved with threepoint functions is the computation of the three-point function of the stress-energy tensor $T_{i j}(\vec{x})$. Unlike the other threepoint functions that are determined by the conformal symmetry almost completely (usually up to one constant), the three-point function of $T_{i j}(\vec{x})$ in general dimension $d$ admits five independent conformally covariant forms, two of them being fixed by the gauge-theory conservation law $\partial_{i} T_{i j}(\vec{x})$ $=0$ [29]. Three constants undetermined neither conformal symmetry nor the conservation law might be computed from the AdS supergravity and confronted with their free-field counterparts. This obviously provides a further nontrivial test for the AdS-CFT correspondence.

In this paper we therefore address the problem of computing the three-point function of the stress-energy tensor in the $d$-dimensional CFT from $\mathrm{AdS}_{d+1}$ gravity.

According to the AdS-CFT conjecture the CFT current of conformal weight zero coupled to the stress-energy tensor of conformal dimension $d$ is extended to the interior of the AdS space as the on-shell graviton field. In comparison with three-point functions of other gauge-invariant composite op-

\footnotetext{
*Email address: arut@genesis.mi.ras.ru

†Email address: frolov@bama.ua.edu
}

erators computation of the three-point function of $T_{i j}(\vec{x})$ is complicated by two things. First to clear up the infrared divergences of the gravity action, one can introduce the boundary of the AdS space. The Hamiltonian formulation [13] of the AdS-CFT correspondence then naturally requires additional boundary terms [8], so that the pure gravity action is not given only by the standard Einstein-Hilbert term. It is worth stressing that the account of the boundary terms is absolutely necessary since they provide the fulfillment of the Ward identities in the boundary CFT. The second thing is that after introducing the boundary terms the gravity action loses its manifest conformal as well as gauge invariance.

In our computation of the three-point function we account the boundary terms in the following way. We start with the standard Einstein-Hilbert term (with the cosmological constant) and decompose it up to the cubic order in metric perturbation $h_{\mu \nu}$. Removing all terms linear in second derivatives as well as all total derivative terms we are left with an action that according to [13] differs from the action one should use in the AdS-CFT correspondence only by terms that do not contribute to the Green functions. Then, by the on-shell perturbation theory, one may find that the contribution of the quadratic terms to the value of the three-point is exactly zero. Thus, the remaining action is just the sum of the cubic bulk and the boundary (noncovariant) terms, the latter arise due to the removal procedure. Fortunately, the cubic boundary terms do not contribute to the value of the three-point function and by this reason can be disregarded. The bulk term is manifestly covariant with respect to the AdS isometries as well as on-shell gauge symmetry and that apparently solves the second difficulty.

Choosing the covariant gauge we then explicitly compute the remaining bulk integral and get the three-point function. For the physically most interesting case $d=4$ we realize that the coefficients of the conformal tensors of the three-point function are exactly the same as the ones found by the freefield computations. This is obviously in agreement with the earlier results by $[12,30,31]$, whose essence is that in four dimensions the superconformal symmetry is powerful 
enough to protect some three-point functions in $\mathcal{N}=4$ YangMills against quantum corrections.

As to the other cases of particular interest $d=3$ and $d$ $=6$, at the moment we are unaware of the gauge-theory calculations and may suggest that our results give the value of the corresponding three-point function in the theories of the strongly coupled superconformal scalar and $(2,0)$ tensor multiplets, respectively.

The paper is organized as follows. In Sec. II we define the gravity action one should use in the AdS-CFT correspondence and obtain its decomposition up to cubic order in metric perturbation. In Sec. III we compute the on-shell value of the gravity action in the de Donder gauge and obtain the three-point function. We then write down explicitly the coefficients of the conformal tensors occurring in the threepoint function in dimensions $d=2,3,4,5,6$ and comment on the most interesting cases. Some details of the calculation are collected in the Appendix.

\section{GRAVITY ACTION AT THIRD ORDER OF PERTURBATION}

We begin by fixing the basic notation. Let $\mathcal{M}$ be a $(d$ +1 )-dimensional manifold with a $d$-dimensional boundary. Throughout the paper the indices $\mu, \nu, \ldots$ run the set $0, \ldots, d$, while $i, j, \ldots$ are reserved for the $d$-dimensional boundary and take values $1, \ldots, d$. The coordinates $x_{\mu}$ are then split as $x_{\mu}=\left(x_{0}, \vec{x}\right)$ with $\vec{x}=\left\{x_{i}\right\}$. Let $\mathcal{M}$ be also supplied with a positive signature metric $G_{\mu \nu}$.

We will deal with the Euclidean version of the $\operatorname{AdS}_{d+1}$ space that is described as the upper half space $\mathcal{M}=\left\{x_{i}\right.$ $\left.\in \mathbf{R} ; \quad x_{0}>0\right\}$ endowed with the metric

$$
d s^{2}=\frac{1}{x_{0}^{2}} d x^{\mu} d x^{\mu} .
$$

The boundary of the $\operatorname{AdS}_{d+1}$ space is at $x_{0}=0$ and can be identified with the Euclidean space. Since the boundary is infinitely distant from any interior point, the gravity action on the AdS background suffers from infrared divergences. A natural regularization is then provided by setting the boundary of $\mathrm{AdS}_{d+1}$ space at $x_{0}=\varepsilon$ and considering the part with $x_{0} \geqslant \varepsilon$. The physical fields are required to vanish when $x_{0}$ $\rightarrow \infty$.

The total gravity action $\mathbf{S}$ one should use for computing gauge theory correlation functions is given by the sum

$$
\mathbf{S}=S+S^{(1)}+S^{(2)}
$$

of the standard Einstein-Hilbert term [with the cosmological constant $\left.\lambda=\frac{1}{2} d(d-1)\right]$

$$
S=\int \sqrt{G}(R-2 \lambda)
$$

and two boundary terms $S^{(1)}$ and $S^{(2)}$ [8]. Explicitly, $S^{(1)}$ is the Gibbons-Hawking term [32]

$$
S^{(1)}=2 \int_{\partial M} d^{d} x \sqrt{\bar{G}} K,
$$

where $K$ is the trace of the fundamental form on the boundary and $\bar{G}$ is the determinant of the induced metric. The second boundary term $S^{(2)}$ is a term proportional to the volume of the boundary:

$$
S^{(2)}=2(1-d) \int_{\partial M} d^{d} x \sqrt{\bar{G}} .
$$

As was shown in [13], adding the boundary terms is equivalent to removing from the bulk action (2) all terms linear in second derivatives and all total derivative terms. The gravity action obtained in such a way differs from Eq. (1) only by terms which do not contribute to Green functions. Therefore, we can restrict our attention to considering the bulk term.

If we assume $g_{\mu \nu}$ to be the background AdS metric and perturb $G_{\mu \nu}$ near the background value: $G_{\mu \nu}=g_{\mu \nu}+h_{\mu \nu}$, then equations of motion $R_{\mu \nu}=-d g_{\mu \nu}$ up to the second order in $h_{\mu \nu}$ can be written as follows:

$$
L_{\mu \nu}=V_{\mu \nu}
$$

where two tensors

$$
\begin{aligned}
L_{\mu \nu}= & \nabla^{\rho} \nabla_{\rho} h_{\mu \nu}+\nabla_{\mu} \nabla_{\nu} h-\nabla_{\mu} \nabla^{\rho} h_{\nu \rho}-\nabla_{\nu} \nabla^{\rho} h_{\mu \rho} \\
& +2\left(h_{\mu \nu}-g_{\mu \nu} h\right),
\end{aligned}
$$

and

$$
\begin{aligned}
V_{\mu \nu}= & -\nabla_{\rho}\left[h^{\rho \sigma}\left(\nabla_{\mu} h_{\nu \sigma}+\nabla_{\nu} h_{\mu \sigma}-\nabla_{\sigma} h_{\mu \nu}\right)\right] \\
& +\nabla_{\nu}\left(h^{\rho \sigma} \nabla_{\mu} h_{\rho \sigma}\right)+\frac{1}{2}\left(\nabla_{\mu} h_{\nu \rho}+\nabla_{\nu} h_{\mu \rho}-\nabla_{\rho} h_{\mu \nu}\right) \nabla^{\rho} h \\
& -\frac{1}{2} \nabla_{\mu} h_{\rho \sigma} \nabla_{\nu} h^{\rho \sigma}+\nabla_{\rho} h_{\mu \sigma} \nabla^{\rho} h_{\nu}^{\sigma}-\nabla_{\sigma} h_{\mu \rho} \nabla^{\rho} h_{\nu}^{\sigma}
\end{aligned}
$$

were introduced. Here the covariant derivatives are taken with respect to the background metric.

Introduce the notation

$$
\begin{aligned}
R_{\mu \nu} & =\dot{R}_{\mu \nu}+R_{\mu \nu}^{(1)}+R_{\mu \nu}^{(2)}+\cdots \\
& =\dot{R}_{\mu \nu}+\delta R_{\mu \nu}+\frac{1}{2 !} \delta^{(2)} R_{\mu \nu}+\cdots
\end{aligned}
$$

for decomposition of the Ricci tensor around the background $\dot{R}_{\mu \nu}$ and the analogous one for decomposition of the curvature.

Now we are ready to analyze gravity action (1) up to the third order in metric perturbation. We start with working out decomposition of Eq. (2):

$$
S=\dot{S}+\delta S+\frac{1}{2 !} \delta^{(2)} S+\frac{1}{3 !} \delta^{(3)} S+\cdots
$$


Computing the first variation $\delta S$ of Eq. (2) one then represents it in the form

$$
\delta S[G, h]=-\int_{M} \sqrt{G}\left(R_{\mu \nu}-\frac{1}{2} G_{\mu \nu} R+\lambda G_{\mu \nu}\right) h^{\mu \nu}+T,
$$

where $T$ is the following boundary term:

$$
T=\int_{M} \sqrt{G} \nabla_{\mu}\left(\nabla_{\nu} h^{\mu \nu}-\nabla^{\mu} h\right)=-\int_{\partial M} \sqrt{G}\left(\nabla_{\mu} h^{0 \mu}-\partial^{0} h\right) .
$$

In Eq. (9) we consider $\delta S[G, h]$ as the variation of $S[G]$ at a "point" $G_{\mu \nu}$, i.e., we do not assume the metric $G_{\mu \nu}$ to be equal to its background value. Now the simple algorithm to find decomposition (8) is to consider the successive variations of $\delta S[G, h] .{ }^{1}$ Since we are interested in decomposition of the total action (1) we can omit the total derivative term $T$.

Thus, varying the terms in parentheses in Eq. (9) and reducing the result to the background we get

$$
R_{\mu \nu}^{(1)}-\frac{1}{2} g_{\mu \nu}^{(1)} \dot{R}-\frac{1}{2} g_{\mu \nu} R^{(1)}+\lambda g_{\mu \nu}^{(1)}=-\frac{1}{2}\left(L_{\mu \nu}-\frac{1}{2} g_{\mu \nu} L\right),
$$

where the relation $R_{\mu \nu}^{(1)}+d h_{\mu \nu}=-\frac{1}{2} L_{\mu \nu}$ was used.

For the second variation we find

$$
\begin{aligned}
\delta^{(2)} R_{\mu \nu}-\frac{1}{2} g_{\mu \nu} \delta^{(2)} R-\frac{1}{2} \delta^{(1)} g_{\mu \nu} \delta^{(1)} R \\
\quad=2\left(R_{\mu \nu}^{(2)}-\frac{1}{2} g_{\mu \nu}\left(g^{\rho \sigma} R_{\rho \sigma}^{(2)}\right)\right)-\frac{1}{2} g_{\mu \nu} h^{\rho \sigma} L_{\rho \sigma}+\frac{1}{2} h_{\mu \nu} L .
\end{aligned}
$$

Here $R_{\mu \nu}^{(2)}-\frac{1}{2} g_{\mu \nu}\left(g^{\rho \sigma} R_{\rho \sigma}^{(2)}\right)=\frac{1}{2}\left(V_{\mu \nu}-\frac{1}{2} g_{\mu \nu} V\right)$, where $\quad V$ $=V_{\mu}^{\mu}$.

With these formulas at hand it is now easy to find the action (2) up to the third order in $h_{\mu \nu}$ :

$$
\begin{aligned}
S= & \dot{S}+\int_{M} \sqrt{g}\left[\frac{1}{4}\left(L_{\mu \nu}-\frac{1}{2} g_{\mu \nu} L\right) h^{\mu \nu}-\frac{1}{6}\left(V_{\mu \nu}-\frac{1}{2} g_{\mu \nu} V\right)\right. \\
& \left.\times h^{\mu \nu}-\frac{1}{6}\left(\frac{1}{8} h^{2} L-\frac{3}{4} h h^{\mu \nu} L_{\mu \nu}+h^{\mu \rho} h_{\rho}^{\nu} L_{\mu \nu}\right)\right],
\end{aligned}
$$

where we again omitted nonessential total derivative terms. Note that the last term in the integrand coincides with the third-order term in the decomposition of $\sqrt{G}$, in which one tensor $h_{\mu \nu}$ is replaced by $L_{\mu \nu}$. The action (10) depends on second derivative terms. To remove these terms one should add to Eq. (10) total derivative terms which can be easily found by using explicit expressions (6) and (7) for $L$ and $V$, respectively. A simple consideration then shows that the quadratic terms in the resulting action do not contribute to the three-point Green function.

\footnotetext{
${ }^{1}$ The covariant derivatives in $T$ are also with respect to the metric $G_{\mu \nu}$.
}

Thus, we see that to find the three-point function we need to compute the on-shell value of $\mathbf{S}$ which is given by

$$
\mathbf{S}=-\frac{1}{6} \int_{M} \sqrt{g}\left(V_{\mu \nu}-\frac{1}{2} g_{\mu \nu} V\right) h^{\mu \nu}+\text { cubic. }
$$

Here $h_{\mu \nu}$ is a solution to the linearized equation of motion: $L_{\mu \nu}[h]=0$ and cubic refers to the unwritten explicitly total derivative terms of the cubic order. Since these terms may deliver only a local contribution to the value of the threepoint function, in what follows we disregard them.

\section{THREE-POINT GREEN FUNCTION}

The radiation gauge for the AdS gravity that is effectively used for computing the two-point Green function of the stress-energy tensor in the boundary CFT obviously breaks the invariance of the gravity action under isometries. For the three-point function this fact leads to severe difficulties in computing the bulk integrals. Thus, to handle the problem we choose the covariant gauge of the de Donder type:

$$
\nabla_{\mu}\left(h_{\nu}^{\mu}-\frac{1}{2} \delta_{\nu}^{\mu} h\right)=0
$$

In this gauge the solution of the linearized equations of motion reads as [8]

$$
\begin{aligned}
h_{\mu}^{\nu}\left(x_{0}, \vec{x}\right)= & \kappa_{G} \int d^{d} y \mathcal{K}(x, \vec{y}) J_{\mu}^{i}(x-\vec{y}) \\
& \times J_{j}^{\nu}(x-\vec{y}) \mathcal{E}_{i j, k l} h_{k l}(\vec{y}),
\end{aligned}
$$

where

$$
J_{\mu}^{\nu}(x)=\delta_{\mu}^{\nu}-2 \frac{x_{\mu} x^{\nu}}{|x|^{2}}, \quad \mathcal{K}(x, \vec{y})=\frac{x_{0}^{d}}{\left[x_{0}^{2}+(\vec{x}-\vec{y})^{2}\right]^{d}},
$$

the coefficient $k_{G}=(d+1) /(d-1) \Gamma(d) / \pi^{d / 2} \Gamma(d / 2), h_{l}^{k}(\vec{y})$ represents the boundary data of the graviton and $\mathcal{E}_{i j, k l}$ is the traceless symmetric projector:

$$
\mathcal{E}_{i j, k l}=\frac{1}{2}\left(\delta_{i k} \delta_{j l}+\delta_{i l} \delta_{k j}\right)-\frac{1}{d} \delta_{i j} \delta_{k l}
$$

Note that tensor $h_{\mu}^{\nu}$ has the vanishing trace.

In the de Donder gauge the remaining bulk term of the on-shell action (11) can be represented in the following form most suitable for further computations: 


$$
\begin{aligned}
\mathbf{S}= & -\frac{1}{6} \int_{M} \sqrt{-g}\left(V_{\mu \nu}-\frac{1}{2} g_{\mu \nu} V\right) h^{\mu \nu} \\
= & \frac{1}{6} \int_{M} \sqrt{-g}\left(\nabla_{\sigma}\left(h^{\mu \nu} h_{\mu \rho} \nabla^{\rho} h_{\nu}^{\sigma}\right)-\frac{1}{2} \nabla_{\mu}\left(h^{\mu \nu} h^{\rho \sigma} \nabla_{\nu} h_{\rho \sigma}\right)\right. \\
& \left.-\nabla_{\sigma}\left(h_{\mu}^{\sigma} h_{\nu}^{\rho} \nabla_{\rho} h^{\mu \nu}\right)\right)-\frac{1}{4} \int_{M} \sqrt{-g}\left(\nabla_{\sigma} \nabla_{\rho} h_{\mu \nu} h^{\mu \nu} h^{\rho \sigma}\right. \\
& -2 \nabla_{\sigma} \nabla_{\rho} h^{\mu \nu} h_{\mu}^{\rho} h_{\nu}^{\sigma}+\frac{2}{3} h_{\mu}^{\nu} \nabla_{\rho} h_{\sigma}^{\mu} \nabla^{\rho} h_{\nu}^{\sigma} \\
& \left.-\frac{2}{3}(d+1) h_{\mu}^{\nu} h_{\sigma}^{\mu} h_{\nu}^{\sigma}\right),
\end{aligned}
$$

where we used the explicit form of $V_{\mu \nu}$ and the vanishing of $h_{\mu}^{\mu}$.

Again omitting the total derivatives being the cubic order boundary terms we see that the on-shell action in the de Donder gauge is essentially given by the bulk integral

$$
\begin{aligned}
\mathbf{S}= & -\frac{1}{4} \int_{M} \sqrt{-g}\left(\nabla_{\sigma} \nabla_{\rho} h_{\mu \nu} h^{\mu \nu} h^{\rho \sigma}-2 \nabla_{\sigma} \nabla_{\rho} h^{\mu \nu} h_{\mu}^{\rho} h_{\nu}^{\sigma}\right. \\
& \left.+\frac{2}{3} h_{\mu}^{\nu} \nabla_{\rho} h_{\sigma}^{\mu} \nabla^{\rho} h_{\nu}^{\sigma}-\frac{2}{3}(d+1) h_{\mu}^{\nu} h_{\sigma}^{\mu} h_{\nu}^{\sigma}\right) .
\end{aligned}
$$

By using the equation of motion that in the covariant gauge reads as $\nabla_{\rho} \nabla^{\rho} h_{\mu \nu}=-2 h_{\mu \nu}$ we then rewrite Eq. (15) in the form

$$
\begin{aligned}
\mathbf{S}= & \frac{1}{4} \int_{M} \sqrt{-g}\left(2 \nabla_{\sigma} \nabla_{\rho} h^{\mu \nu} h_{\mu}^{\rho} h_{\nu}^{\sigma}-\nabla_{\sigma} \nabla_{\rho} h_{\mu \nu} h^{\mu \nu} h^{\rho \sigma}\right. \\
& \left.+\frac{2}{3} d h_{\mu}^{\nu} h_{\sigma}^{\mu} h_{\nu}^{\sigma}\right) .
\end{aligned}
$$

The computation of Eq. (16) is a rather combersome but purely technical task that can be performed by the inversion method of [7]. Before plugging into details we make some comments about the relation between the bulk and the boundary gauge transformations. The symmetry group of action (16) is now reduced to the gauge transformations that preserves the de Donder gauge. This group of residual gauge transformations is generated by vectors $\xi^{\mu}$ obeying an equation $\nabla_{\rho} \nabla^{\rho} \xi_{\mu}-d \xi_{\mu}=0$. Explicitly, the solution satisfying the gauge $\nabla_{\mu} \xi^{\mu}=0$ reads as

$$
\xi^{\mu}\left(x_{0}, \vec{x}\right)=\kappa_{v} \int d^{d} y \frac{x_{0}^{d+2}}{\left[x_{0}^{2}+(\vec{x}-\vec{y})^{2}\right]^{d+1}} J_{i}^{\mu}(x-\vec{y}) \xi^{i}(\vec{y})
$$

$$
\kappa_{v}=\frac{d+1}{d} \frac{2 \Gamma(d)}{\pi^{d / 2} \Gamma(d / 2)}
$$

where the coefficient $\kappa_{v}$ is fixed by requiring $\xi^{i}\left(x_{0}, \vec{x}\right)$ $\rightarrow \xi^{i}(\vec{x})$ when $x_{0} \rightarrow 0$. In particular, the component $\xi^{0}$ is

$$
\xi^{0}\left(x_{0}, \vec{x}\right)=\frac{\kappa_{v}}{d+1} \int d^{d} y \frac{x_{0}^{d+3}}{\left[x_{0}^{2}+(\vec{x}-\vec{y})^{2}\right]^{d+1}} \partial_{i} \xi^{i}(\vec{y})
$$

One may see that on the boundary $(\varepsilon \rightarrow 0)$ the residual gauge transformations are reduced to

$$
\delta h_{i}^{j}=\partial_{i} \xi^{j}+\partial_{j} \xi^{i}-\frac{2}{d} \delta_{i j}\left(\partial_{k} \xi^{k}\right)
$$

i.e., to the usual gauge transformations of a traceless symmetric tensor. In other words, the transformations (18) of the boundary data can always be prolonged to the bulk gauge fields, which preserve the de Donder gauge. We, therefore, expect the three-point function $T_{i j, k l, m n}(\vec{x}, \vec{y}, \vec{z})$ $=\left\langle T_{i j}(\vec{x}) T_{k l}(\vec{y}) T_{m n}(\vec{z})\right\rangle$ of the stress-energy tensor $T_{i j}$ to obey the conservation law

$$
\partial_{i} T_{i j, k l, m n}(\vec{x}, \vec{y}, \vec{z})=0, \quad \text { for noncoincident } \vec{x}, \vec{y} \text { and } \vec{z}
$$

As was already mentioned in the Introduction in arbitrary dimension $d>3$ there are five independent conformal tensors occuring in the expression for the three-point function of the stress-energy tensor. The conservation law then fixes the value of two from five coefficients. It is clear that the threepoint function defined by $S$ is conformally covariant and the only reason to find its explicit expression is to make comparison of the coefficients of conformal tensors to the ones found on the gauge theory side.

Thus, substituting Eq. (13) in Eq. (16) we see that according to the AdS-CFT prescription $[2,3]$ the three-point function is defined as ${ }^{2}$

$$
T_{i j, k l, m n}(\vec{x}, \vec{y}, \vec{z})=8 \sum I_{i j, k l, m n}(\vec{x}, \vec{y}, \vec{z})
$$

where the sum is taken over all possible permutations of sets of indices and points $(i j, \vec{x}),(k l, \vec{y})$, and $(m n, \vec{z})$ of the following tensor:

\footnotetext{
${ }^{2}$ We assume that the coupling of $T_{i j}(\vec{x})$ with $h_{i j}(\vec{x})$ on the boundary of $\operatorname{AdS}_{d+1}$ is given by $\int d^{d} x\left[\frac{1}{2} T_{i j}(\vec{x}) h_{i j}(\vec{x})\right]$ and this explains the number 8 in Eq. (19). Later on we show that this coupling also leads to the correct Ward identity.
} 


$$
\begin{aligned}
I_{i j, k l, m n}(\vec{x}, \vec{y}, \vec{z})= & 2 \kappa_{G}^{3} \mathcal{E}_{i j, i^{\prime} j^{\prime}} \mathcal{E}_{k l, k^{\prime} l^{\prime}} \mathcal{E}_{m n, m^{\prime} n^{\prime}} \int \frac{d^{d+1} \omega}{\omega_{0}^{d+1}} \mathcal{K}(\omega, \vec{y}) \mathcal{K}(\omega, \vec{z}) \times\left[2 \nabla^{\sigma} \nabla_{\rho}\left(\mathcal{K} J_{i^{\prime}}^{\mu} J_{\nu}^{j^{\prime}}\right)(\omega-\vec{x})\left(J_{\mu}^{k^{\prime}} J_{l^{\prime}}^{\rho}\right)(\omega-\vec{y})\left(J_{\sigma}^{m^{\prime}} J_{n^{\prime}}^{\nu}\right)\right. \\
& \times(\omega-\vec{z})-\nabla_{\sigma} \nabla^{\rho}\left(\mathcal{K} J_{i^{\prime}}^{\mu} J_{\nu}^{j^{\prime}}\right)(\omega-\vec{x})\left(J_{\mu}^{k^{\prime}} J_{l^{\prime}}^{\nu}\right)(\omega-\vec{y})\left(J_{\rho}^{m^{\prime}} J_{n^{\prime}}^{\sigma}\right)(\omega-\vec{z}) \\
& \left.+\frac{2 d}{3}\left(\mathcal{K} J_{i^{\prime}}^{\mu} J_{\nu}^{j^{\prime}}\right)(\omega-\vec{x})\left(J_{\mu}^{k^{\prime}} J_{l^{\prime}}^{\rho}\right)(\omega-\vec{y})\left(J_{\rho}^{m^{\prime}} J_{n^{\prime}}^{\nu}\right)(\omega-\vec{z})\right]
\end{aligned}
$$

Recall that here and in what follows the bulk (boundary) indices are contracted with respect to the AdS metric (Euclidean) and, therefore, only their positions matter. Note that the tensor $I_{i j, k l, m n}$ itself is not conformally covariant.

Following the method of [7] we now set in Eq. (20) $\vec{x}=0$ and perform the change of variables $\omega_{\mu}^{\prime}=\omega_{\mu} / \omega^{2}$ and $x_{i}^{\prime}$ $=x_{i} / x^{2}$. This is just the inversion transformation under which the derivatives $\nabla_{\mu}$ transform covariantly. In particular,

$$
\nabla_{\rho}\left[\mathcal{K}(\omega, \vec{x}) J_{\mu}^{i}(\omega-\vec{x}) J_{j}^{\nu}(\omega-\vec{x})\right]=\left|\omega^{\prime}\right|^{2} J_{\mu}^{\lambda}\left(\omega^{\prime}\right) J_{\beta}^{\nu}\left(\omega^{\prime}\right) J_{\rho}^{\sigma}\left(\omega^{\prime}\right) \nabla_{\sigma}^{\prime}\left[\mathcal{K}\left(\omega^{\prime}, \vec{x}^{\prime}\right) J_{\lambda}^{a}\left(\omega^{\prime}-\vec{x}^{\prime}\right) J_{b}^{\beta}\left(\omega^{\prime}-\vec{x}^{\prime}\right)\right] \frac{J_{a}^{i}(\vec{x}) J_{j}^{b}(\vec{x})}{|x|^{2 d}}
$$

and

$$
\nabla_{\rho}\left[\mathcal{K}(\omega, 0) J_{\mu}^{i}(\omega) J_{j}^{\nu}(\omega)\right]=\left|\omega^{\prime}\right|^{2} J_{\mu}^{\lambda}\left(\omega^{\prime}\right) J_{\beta}^{\nu}\left(\omega^{\prime}\right) J_{\rho}^{\sigma}\left(\omega^{\prime}\right) \nabla_{\sigma}^{\prime}\left(\left(\omega_{0}^{\prime}\right)^{d} \delta_{\lambda}^{i} \delta_{j}^{\beta}\right)
$$

where the covariant derivatives $\nabla_{\mu}^{\prime}$ are with respect to to the connection

$$
\Gamma_{\rho \sigma}^{\prime \mu}\left(\omega^{\prime}\right)=-\frac{1}{\omega_{0}{ }^{\prime}}\left(\delta_{\rho}^{0} \delta_{\mu \sigma}+\delta_{\sigma}^{0} \delta_{\mu \rho}-\delta_{\mu}^{0} \delta_{\rho \sigma}\right)
$$

Thus, after substituting the change of variables all internal Jacobians depending on the variable $\omega$ alone cancel against each other and one is left with the following expression:

$$
\begin{aligned}
I_{i j, k l, m n}(0, \vec{y}, \vec{z})= & 2 \kappa_{G}^{3} \mathcal{E}_{i j, i^{\prime} j^{\prime}} \frac{\mathcal{I}_{k l, k^{\prime} l^{\prime}}(\vec{y})}{|y|^{2 d}} \frac{\mathcal{I}_{m n, m^{\prime} n^{\prime}}(\vec{z})}{|z|^{2 d}} \int \frac{d^{d+1} \omega}{\omega_{0}} \mathcal{K}\left(\omega, \vec{y}^{\prime}\right) \mathcal{K}\left(\omega, \vec{z}^{\prime}\right) \times\left[-\frac{4 d}{3}\left(J_{i^{\prime}}^{k^{\prime}} J_{l^{\prime}}^{s}\right)\left(\omega-\vec{y}^{\prime}\right)\left(J_{s}^{m^{\prime}} J_{n^{\prime}}^{j^{\prime}}\right)\left(\omega-\vec{z}^{\prime}\right)\right. \\
& +2\left(d^{2}+\frac{d}{3}+2\right)\left(J_{i^{\prime}}^{k^{\prime}} J_{l^{\prime}}^{0}\right)\left(\omega-\vec{y}^{\prime}\right)\left(J_{j^{\prime}}^{m^{\prime}} J_{n^{\prime}}^{0}\right)\left(\omega-\vec{z}^{\prime}\right)-\left(d^{2}-d-2\right)\left(J_{i^{\prime}}^{k^{\prime}} J_{l^{\prime}}^{j^{\prime}}\right)\left(\omega-\vec{y}^{\prime}\right)\left(J_{0}^{m^{\prime}} J_{n^{\prime}}^{0}\right)\left(\omega-\vec{z}^{\prime}\right) \\
& \left.+2 d\left(J_{0}^{k^{\prime}} J_{l^{\prime}}^{0}\right)\left(\omega-\vec{y}^{\prime}\right)\left(J_{i^{\prime}}^{m^{\prime}} J_{n^{\prime}}^{j^{\prime}}\right)\left(\omega-\vec{z}^{\prime}\right)\right]
\end{aligned}
$$

where the concise notation $\mathcal{I}_{i j, k l}(\vec{x})=\mathcal{E}_{i j, i^{\prime} j^{\prime}}\left(J_{i^{\prime} k} J_{j^{\prime} l}\right)(\vec{x})$ was introduced.

In view of Eq. (19) it is further more convenient to deal with the integral $I_{i j, k l, m n}^{\text {sym }}$ being the symmetrization of Eq. (21) with respect to $(k l, \vec{y})$ and $(m n, \vec{z})$ :

$$
I_{i j, k l, m n}^{\mathrm{sym}}(0, \vec{y}, \vec{z})=I_{i j, k l, m n}(0, \vec{y}, \vec{z})+I_{i j, m n, k l}(0, \vec{z}, \vec{y}) .
$$

The computation of $I^{\mathrm{sym}}$ is sketched in the Appendix and below we present the result 


$$
\begin{aligned}
I_{i j, k l, m n}^{\mathrm{sym}}(0, \vec{y}, \vec{z})= & 2 \pi^{d / 2} \kappa_{G}^{3} \frac{\Gamma(d / 2)[\Gamma(d / 2+1)]^{2}}{(d+1)^{2} \Gamma(d-1) \Gamma(d+2)} \mathcal{E}_{i j, i^{\prime} j^{\prime}} \frac{\mathcal{I}_{k l, k^{\prime} l^{\prime}}(\vec{y})}{|y|^{2 d}} \frac{\mathcal{I}_{m n, m^{\prime} n^{\prime}}(\vec{z})}{|z|^{2 d}} \frac{1}{|t|^{d}} \\
& \times\left\{a_{1} \mathcal{E}_{i^{\prime} j^{\prime}, a b} \mathcal{E}_{k^{\prime} l^{\prime}, a c} \mathcal{E}_{m^{\prime} n^{\prime}, b c}+a_{2} \mathcal{E}_{i^{\prime} j^{\prime}, a b} \mathcal{E}_{k^{\prime} l^{\prime}, a c} \mathcal{E}_{m^{\prime} n^{\prime}, b d} \frac{t_{c} t_{d}}{t^{2}}\right. \\
& +a_{3}\left[\mathcal{E}_{k^{\prime} l^{\prime}, a b} \mathcal{E}_{m^{\prime} n^{\prime}, b c} \mathcal{E}_{i^{\prime} j^{\prime}, a d}+\left(k^{\prime} l^{\prime}\right) \rightarrow\left(m^{\prime} n^{\prime}\right)\right] \frac{t_{c} t_{d}}{t^{2}}+a_{4}\left[\mathcal{E}_{k^{\prime} l^{\prime}, i^{\prime} j^{\prime}}\left(\frac{t_{m^{\prime}} t_{n^{\prime}}}{t^{2}}-\frac{1}{d} \delta_{m^{\prime} n^{\prime}}\right)+\left(k^{\prime} l^{\prime}\right) \rightarrow\left(m^{\prime} n^{\prime}\right)\right] \\
& +a_{5} \mathcal{E}_{k^{\prime} l^{\prime}, m^{\prime} n^{\prime}}\left(\frac{t^{\prime} t_{j^{\prime}}}{t^{2}}-\frac{1}{d} \delta_{i^{\prime} j^{\prime}}\right)+a_{6}\left[\mathcal{E}_{k^{\prime} l^{\prime}, a b} \mathcal{E}_{i^{\prime} j^{\prime}, a c}\left(\frac{t_{m^{\prime}} t_{n^{\prime}}}{t^{2}}-\frac{1}{d} \delta_{m^{\prime} n^{\prime}}\right)+\left(k^{\prime} l^{\prime}\right) \rightarrow\left(m^{\prime} n^{\prime}\right)\right] \frac{t_{b} t_{c}}{t^{2}} \\
& \left.+a_{7} \mathcal{E}_{k^{\prime} l^{\prime}, a b} \mathcal{E}_{m^{\prime} n^{\prime}, a c}\left(\frac{t_{i^{\prime}} t_{j^{\prime}}}{t^{2}}-\frac{1}{d} \delta_{i^{\prime} j^{\prime}}\right) \frac{t_{b} t_{c}}{t^{2}}+a_{8}\left(\frac{t_{i^{\prime}} t_{j^{\prime}}}{t^{2}}-\frac{1}{d} \delta_{i^{\prime} j^{\prime}}\right)\left(\frac{t_{k^{\prime}} t_{l^{\prime}}}{t^{2}}-\frac{1}{d} \delta_{k^{\prime} l^{\prime}}\right)\left(\frac{t_{m^{\prime}} t_{n^{\prime}}}{t^{2}}-\frac{1}{d} \delta_{m^{\prime} n^{\prime}}\right)\right\} .
\end{aligned}
$$

In the last formula the Latin indices $(a, b, c, d)$ are used to distinguish the boundary summation indices and the variable $t_{i}$ $=z_{i}^{\prime}-y_{i}^{\prime}$. The coefficients $a_{i}, i=1, \ldots, 8$ are expressed through the constants $a_{i}^{(k)}, k=1,2,3$ found in the Appendix by the following formula:

$$
a_{i}=-\frac{4 d}{3} a_{i}^{(1)}+2\left(d^{2}+\frac{d}{3}+2\right) a_{i}^{(2)}-\left(d^{2}-3 d-2\right) a_{i}^{(3)} .
$$

If we now restore the $\vec{x}$ dependence, the variable $t_{i}$,

$$
t_{i}=(z-x)_{i}^{\prime}-(y-x)_{i}^{\prime}=\frac{(x-y)_{i}}{(\vec{x}-\vec{y})^{2}}-\frac{(x-z)_{i}}{(\vec{x}-\vec{z})^{2}}
$$

turns into the conformal vector $X_{i}: t_{i}=-X_{i}$ with a remarkable property to transform covariantly $X_{i} \rightarrow X_{i}^{\prime}$ under the inversion $x_{i}^{\prime}=x_{i} / x^{2}[29]$ :

$$
X_{i}^{\prime}=x^{2} J_{i j}(\vec{x}) X_{j}
$$

Then by using the two identities

$$
J_{i j}(\vec{x}-\vec{z}) Z_{j}=-\frac{(\vec{x}-\vec{y})^{2}}{(\vec{z}-\vec{y})^{2}} X_{j}, \quad J_{i k}(\vec{x}-\vec{z}) J_{k j}(\vec{z}-\vec{y})=J_{i j}(\vec{x}-\vec{y})+2(\vec{x}-\vec{y})^{2} X_{i} Y_{j},
$$

one may finally represent the three-point function (19) in the form

$$
\begin{aligned}
T_{i j, k l, m n}= & \frac{1}{|\vec{x}-\vec{y}|^{2 d}|\vec{y}-\vec{z}|^{2 d}|\vec{x}-\vec{z}|^{2 d}}\left\{\mathcal { E } _ { i j , i ^ { \prime } j ^ { \prime } } \mathcal { E } _ { k l , k ^ { \prime } l ^ { \prime } } \mathcal { E } _ { m n , m ^ { \prime } n ^ { \prime } } \left[\mathcal{A} J_{i^{\prime} k^{\prime}}(\vec{x}-\vec{y}) J_{l^{\prime} m^{\prime}}(\vec{y}-\vec{z}) J_{j^{\prime} n^{\prime}}(\vec{z}-\vec{x})+\mathcal{B} J_{i^{\prime} k^{\prime}}(\vec{x}-\vec{y}) J_{j^{\prime} n^{\prime}}\right.\right. \\
& \left.\times(\vec{x}-\vec{z}) Y_{l^{\prime}} Y_{m^{\prime}}(\vec{y}-\vec{z})^{2}+\text { cycl. perm. }\right]+\mathcal{C}\left[\mathcal{I}_{i j, k l}\left(\frac{Z_{n} Z_{m}}{Z^{2}}-\frac{1}{d} \delta_{m n}\right)+\text { cycl. perm }\right]+\mathcal{D}\left[\mathcal{E}_{i j, i^{\prime} j^{\prime}} \mathcal{E}_{k l, k^{\prime} l^{\prime}} X_{i^{\prime}} Y_{k^{\prime}}\right. \\
& \left.\left.\times(\vec{x}-\vec{y})^{2} J_{j^{\prime} l^{\prime}}(\vec{x}-\vec{y})\left(\frac{Z_{m} Z_{n}}{Z^{2}}-\frac{1}{d} \delta_{m n}\right)+\text { cycl. perm. }\right]+\mathcal{E}\left(\frac{X_{i} X_{j}}{X^{2}}-\frac{1}{d} \delta_{i j}\right)\left(\frac{Y_{k} Y_{l}}{Y^{2}}-\frac{1}{d} \delta_{k l}\right)\left(\frac{Z_{m} Z_{n}}{Z^{2}}-\frac{1}{d} \delta_{m n}\right)\right\},
\end{aligned}
$$

where

$$
\begin{aligned}
& \mathcal{A}=3 \Delta_{d} a_{1}, \quad \mathcal{B}=\Delta_{d}\left(2 a_{1}+a_{2}-2 a_{3}\right), \quad \mathcal{C}=\Delta_{d}\left(2 a_{4}+a_{5}\right), \\
& \mathcal{D}=\Delta_{d}\left(4 a_{5}+a_{7}-4 a_{3}-2 a_{6}\right), \quad \mathcal{E}=\Delta_{d}\left(12 a_{5}+6 a_{7}+3 a_{8}\right),
\end{aligned}
$$

and

$$
\Delta_{d}=2 \pi^{d / 2} \kappa_{G}^{3} \frac{\Gamma(d / 2)[\Gamma(d / 2+1)]^{2}}{(d+1)^{2} \Gamma(d-1) \Gamma(d+2)}=\frac{d \Gamma(d)}{2 \pi^{d}(d-1)^{2}} .
$$

As was expected formula (25) is just the conformally cova- 
TABLE I. Coefficients $a_{i}^{(k)}$ for $d=4$.

\begin{tabular}{lcccccccc}
\hline \hline$a_{i}^{(k)}$ & $a_{1}^{(k)}$ & $a_{2}^{(k)}$ & $a_{3}^{(k)}$ & $a_{4}^{(k)}$ & $a_{5}^{(k)}$ & $a_{6}^{(k)}$ & $a_{7}^{(k)}$ & $a_{8}^{(k)}$ \\
\hline$k=1$ & $547 / 21$ & $-1163 / 42$ & $-659 / 42$ & $-25 / 84$ & $-109 / 84$ & $843 / 42$ & $465 / 42$ & $-92 / 7$ \\
$k=2$ & $39 / 14$ & $-78 / 7$ & $-22 / 7$ & $-9 / 7$ & $-2 / 7$ & $66 / 7$ & $24 / 7$ & $-48 / 7$ \\
$k=3$ & $-29 / 21$ & $116 / 21$ & $74 / 21$ & $113 / 21$ & $8 / 21$ & $-74 / 21$ & $-32 / 21$ & $64 / 7$ \\
\hline \hline
\end{tabular}

riant three-point function of the stress-energy tensor in the $d$-dimensional conformal field theory and it involves five independent conformal tensors.

The coefficients $\mathcal{A}, \ldots, \mathcal{E}$ computed for any dimension $d \geqslant 2$ represent our basic result. We first discuss the most interesting case $d=4$. In this case by using Table $\mathrm{I}$ in the Appendix one finds for $\mathcal{A}, \ldots, \mathcal{E}$ the following values:

$$
\begin{gathered}
\mathcal{A}=-\frac{8 \times 128}{9 \pi^{4}}, \quad \mathcal{B}=-\frac{8 \times 392}{9 \pi^{4}}, \quad \mathcal{C}=-\frac{8 \times 184}{9 \pi^{4}}, \\
\mathcal{D}=-\frac{8 \times 472}{9 \pi^{4}}, \quad \mathcal{E}=-\frac{8 \times 304}{9 \pi^{4}} .
\end{gathered}
$$

Now we are ready to confront the coefficients (27) with the ones found by the free-field computations in $\mathcal{N}=4$ SYM. To this end one needs to restore the gravity coupling $g_{G}^{-2}$ that enters as an overall constant in front of the total action $\mathbf{S}$. The value of the coupling constant is fixed by the type-IIB supergravity on the $\operatorname{AdS}_{5} \times S^{5}$ background and is equal to

$$
g_{G}^{2}=\frac{8 \pi^{2}}{N^{2}}
$$

Clearly, to restore the $g_{G}^{2}$ dependence of the three-point function we should multiply Eq. (27) on $g_{G}^{-2}$ and obtain

$$
\mathcal{A}=-\frac{128}{9 \pi^{6}} N^{2}, \quad \mathcal{B}=-\frac{392}{9 \pi^{6}} N^{2}, \quad \mathcal{C}=-\frac{184}{9 \pi^{6}} N^{2}
$$

Recall that the two-point function of $T_{i j}(\vec{x})$ found from the $\mathrm{AdS}_{d+1}$ gravity is given by

$$
\left\langle T_{i j}(\vec{x}), T_{k l}(\vec{y})\right\rangle=\frac{C_{d}}{|\vec{x}-\vec{y}|^{d}} \mathcal{I}_{i j, k l}(\vec{x}-\vec{y})
$$

with the central charge $C_{d}=2 k_{G} d / g_{G}^{2}$. In particular, for $d$ $=4$, one gets $C_{4}=80 / \pi^{2} g_{G}^{2}=\left(10 / \pi^{4}\right) N^{2}$.

In Ref. [33] it was shown that for any four-dimensional free-field theory given by $n_{s}$ scalars, by $n_{f}$ Dirac fermions, and by $n_{v}$ vector fields the coefficients $\mathcal{A}, \mathcal{B}, \mathcal{C}$ are as follows:

$$
\begin{aligned}
& \mathcal{A}=\frac{1}{\pi^{6}}\left(\frac{8}{27} n_{s}-16 n_{v}\right), \\
& \mathcal{B}=-\frac{1}{\pi^{6}}\left(\frac{16}{27} n_{s}+4 n_{f}+32 n_{v}\right), \\
& \mathcal{C}=-\frac{1}{\pi^{6}}\left(\frac{2}{27} n_{s}+2 n_{f}+16 n_{v}\right) .
\end{aligned}
$$

Substituting here the field-theoretical content of the $\mathcal{N}$ $=4 \mathrm{SU}(N) \mathrm{SYM}$ :

$$
n_{s}=6\left(N^{2}-1\right), \quad n_{f}=2\left(N^{2}-1\right), \quad n_{v}=N^{2}-1,
$$

we, thus, arrive at

$$
\begin{gathered}
\mathcal{A}=-\frac{128}{9 \pi^{6}}\left(N^{2}-1\right), \quad \mathcal{B}=-\frac{392}{9 \pi^{6}}\left(N^{2}-1\right), \\
\mathcal{C}=-\frac{184}{9 \pi^{6}}\left(N^{2}-1\right) .
\end{gathered}
$$

The central charge $C_{4}$ can be found by taking into account the Ward identity that relates $C_{4}$ with coefficients $\mathcal{A}, \mathcal{B}, \mathcal{C}$ of the three-point function [29]:

$$
C_{4}=\frac{\pi^{2}}{12}(9 \mathcal{A}-\mathcal{B}-10 \mathcal{C})=\frac{10}{\pi^{4}}\left(N^{2}-1\right) .
$$

It is now obvious that in the large- $N$ limit the coefficients $\mathcal{A}, \mathcal{B}, \mathcal{C}$ and $C_{4}$ of the $\mathcal{N}=4 \mathrm{SU}(N) \mathrm{SYM}$ coincide with the ones found from the $\mathrm{AdS}_{5}$ gravity.

Thus, the coefficients $\mathcal{A}, \mathcal{B}$, and $\mathcal{C}$ of the conformal tensors obtained from the $\mathrm{AdS}_{5}$ gravity and reflecting thereby the strong-coupling behavior of the corresponding gauge theory do not receive corrections to their free-field (oneloop) values. This fact finds a good agreement with the results by $[12,30,31]$. Indeed, the traceless conserved stressenergy tensor occurs in the multiplet of the supercurrent $T$ $=\operatorname{tr}\left(W^{2}\right)$, where $W$ is an analytic superfield describing the $\mathcal{N}=4$ Yang-Mills strength multiplet. In [12] it was checked for the leading components of $T$ being the scalar fields that their three-point functions computed from $\mathrm{AdS}_{5} \times \mathrm{S}^{5}$ supergravity coincide with the one-loop results in the large- $N$ limit. The same conclusion about vanishing of the radiative corrections at order $g^{2}$ was achieved in [30] even for finite $N$. By considering the anomaly in the superconformal symme- 
try, it has been argued in [31] (see also [7]) that the two- and three-point functions of $T$ should actually have a one-loop nature.

Having discussed the four-dimensional case, we now list explicitly the coefficients $\mathcal{A}, \ldots, \mathcal{E}$ for dimensions $d$ $=2,3,5,6$ that can be found from Eq. (24) together with (A1)-(A3).

$d=2$ :

$$
\begin{gathered}
\mathcal{A}=-\frac{32}{\pi^{2}}, \quad \mathcal{B}=-\frac{40}{\pi^{2}}, \quad \mathcal{C}=-\frac{12}{\pi^{2}}, \\
\mathcal{D}=-\frac{32}{\pi^{2}}, \quad \mathcal{E}=-\frac{16}{\pi^{2}} .
\end{gathered}
$$

$d=3$ :

$$
\begin{gathered}
\mathcal{A}=-\frac{3^{4}}{2 \pi^{3}}, \quad \mathcal{B}=-\frac{19 \times 3^{2}}{2 \pi^{3}}, \quad \mathcal{C}=-\frac{11 \times 3^{3}}{2^{3} \pi^{3}}, \\
\mathcal{D}=-\frac{41 \times 3^{2}}{2^{2} \pi^{3}}, \quad \mathcal{E}=-\frac{11 \times 3^{4}}{2^{4} \pi^{3}} .
\end{gathered}
$$

$d=5$ :

$$
\begin{gathered}
\mathcal{A}=-\frac{3 \times 5^{4}}{2^{2} \pi^{5}}, \quad \mathcal{B}=-\frac{303 \times 5^{2}}{2^{2} \pi^{5}}, \quad \mathcal{C}=-\frac{117 \times 5^{3}}{2^{4} \pi^{5}}, \\
\mathcal{D}=-\frac{257 \times 3 \times 5^{2}}{2^{3} \pi^{5}}, \quad \mathcal{E}=-\frac{137 \times 3 \times 5^{3}}{2^{5} \pi^{5}} .
\end{gathered}
$$

$d=6$ :

$$
\mathcal{A}=-\frac{2^{8} \times 3^{5}}{5^{2} \pi^{6}}, \quad \mathcal{B}=-\frac{181 \times 2^{6} \times 3^{3}}{5^{2} \pi^{6}}
$$

$\mathcal{C}=-\frac{59 \times 2^{5} \times 3^{4}}{5^{2} \pi^{6}}, \quad \mathcal{D}=-\frac{119 \times 2^{7} \times 3^{3}}{5^{2} \pi^{6}}, \quad \mathcal{E}=-\frac{2^{7} \times 3^{7}}{5^{2} \pi^{6}}$

In [29] it was shown that the conservation law implies the fulfilment of the following two identities:

$$
\begin{gathered}
\left(d^{2}-4\right) \mathcal{A}+(d+2) \mathcal{B}-4 d \mathcal{C}-2 \mathcal{D}=0, \\
(d-2)(d+4) \mathcal{B}-2 d(d+2) \mathcal{C}+8 \mathcal{D}-4 \mathcal{E}=0 .
\end{gathered}
$$

It is needless to say that coefficients (27) and Eqs. (31)-(34) satisfy both of them. It is, of course, only the check that we have done the computation of Eq. (16) correctly. One should be also aware of the fact that for $d=2$ and $d=3$ the number of linearly independent conformal tensors is reduced to 1 and 2 , respectively [33].

The cases $d=3$ and $d=6$ are of particular interest since according to the AdS-CFT conjecture they correspond to compactifications of the $11 d$ supergravity on $\mathrm{AdS}_{4} \times \mathrm{S}^{7}$ and $\mathrm{AdS}_{7} \times \mathrm{S}^{4}$, respectively. We, therefore, expect that coefficients (32) describe the three-point function of $T_{i j}(\vec{x})$ in the strongly coupled three-dimensional gauge theory of the $\mathcal{N}$ $=8$ conformal scalar multiplet, while Eq. (34) are expected to be found in the six-dimensional gauge theory of the $(0,2)$ tensor multiplet [34-37].

\section{ACKNOWLEDGMENTS}

G.A. would like to thank Professor F. Magri for the kind hospitality at the Dipartimento di Matematica dell' Universita di Milano, where this work was completed. The work of G.A. was supported by the Cariplo Foundation for Scientific Research and in part by the RFBI Grant No. N96-01-00608, and the work of S.F. was supported by the U.S. Department of Energy under Grant No. DE-FG02-96ER40967 and in part by RFBI Grant No. N96-01-00551.

APPENDIX

We sketch here for the reader's convenience some details of computation of integral (22).

Clearly, Eq. (22) can be written as the following sum of the integrals $I^{k}, k=1,2,3$ :

$$
I_{i j, k l, m n}^{\mathrm{sym}}=\frac{\kappa_{G}^{3}}{2} \mathcal{E}_{i j, i^{\prime} j^{\prime}} \frac{\mathcal{I}_{k l, k^{\prime} l^{\prime}}(\vec{y})}{|y|^{2 d}} \frac{\mathcal{I}_{m n, m^{\prime} n^{\prime}}(\vec{z})}{|z|^{2 d}}\left[-\frac{4 d}{3} I_{i^{\prime} j^{\prime}, k^{\prime} l^{\prime}, m^{\prime} n^{\prime}}^{1}+2\left(d^{2}+\frac{d}{3}+2\right) I_{i^{\prime} j^{\prime}, k^{\prime} l^{\prime}, m^{\prime} n^{\prime}}^{2}-\left(d^{2}-d-2\right) I_{i^{\prime} j^{\prime}, k^{\prime} l^{\prime}, m^{\prime} n^{\prime}}^{3}\right],
$$

where $I^{k}$ are given by

$$
\begin{aligned}
I_{i j, k l, m n}^{1}(0, \vec{y}, \vec{z})= & \mathcal{E}_{i j, i^{\prime} j^{\prime}} \mathcal{E}_{k l, k^{\prime} l^{\prime}} \mathcal{E}_{m n, m^{\prime} n^{\prime}} \int \frac{d^{d+1} \omega}{\omega_{0}} \mathcal{K}\left(\omega, \overrightarrow{y^{\prime}}\right) \mathcal{K}\left(\omega, \vec{z}^{\prime}\right)\left(J_{i^{\prime}}^{k^{\prime}} J_{l^{\prime}}^{s}\right)\left(\omega-\vec{y}^{\prime}\right)\left(J_{s}^{m^{\prime}} J_{n^{\prime}}^{j^{\prime}}\right)\left(\omega-\vec{z}^{\prime}\right), \\
I_{i j, k l, m n}^{2}(0, \vec{y}, \vec{z})= & \mathcal{E}_{i j, i^{\prime} j^{\prime}} \mathcal{E}_{k l, k^{\prime} l^{\prime}} \mathcal{E}_{m n, m^{\prime} n^{\prime}} \int \frac{d^{d+1} \omega}{\omega_{0}} \mathcal{K}\left(\omega, \vec{y}^{\prime}\right) \mathcal{K}\left(\omega, \vec{z}^{\prime}\right)\left(J_{i^{\prime}}^{k^{\prime}} J_{l^{\prime}}^{0}\right)\left(\omega-\vec{y}^{\prime}\right)\left(J_{j^{\prime}}^{m^{\prime}} J_{n^{\prime}}^{0}\right)\left(\omega-\vec{z}^{\prime}\right), \\
I_{i j, k l, m n}^{3}(0, \vec{y}, \vec{z})= & \mathcal{E}_{i j, i^{\prime} j^{\prime}} \mathcal{E}_{k l, k^{\prime} l^{\prime}} \mathcal{E}_{m n, m^{\prime} n^{\prime}} \int \frac{d^{d+1} \omega}{\omega_{0}} \mathcal{K}\left(\omega, \overrightarrow{y^{\prime}}\right) \mathcal{K}\left(\omega, \vec{z}^{\prime}\right) \\
& \times \frac{1}{2}\left[\left(J_{j^{\prime}}^{k^{\prime}} J_{l^{\prime}}^{i^{\prime}}\right)\left(\omega-\vec{y}^{\prime}\right)\left(J_{0}^{m^{\prime}} J_{n^{\prime}}^{0}\right)\left(\omega-\vec{z}^{\prime}\right)+\left(J_{0}^{k^{\prime}} J_{l^{\prime}}^{0}\right)\left(\omega-\vec{y}^{\prime}\right)\left(J_{j^{\prime}}^{m^{\prime}} J_{n^{\prime}}^{i^{\prime}}\right)\left(\omega-\vec{z}^{\prime}\right)\right] .
\end{aligned}
$$


Then by using the identities

$$
\begin{aligned}
& \mathcal{E}_{k l, k^{\prime} l^{\prime}}\left(J_{i}^{k^{\prime}} J_{l^{\prime}}^{j}\right)(\omega) \frac{\omega_{0}^{d}}{\left(\omega_{0}^{2}+\vec{\omega}^{2}\right)^{d}}=\mathcal{E}_{i j, k^{\prime} l^{\prime}}\left(\frac{d-1}{d+1} \delta_{i}^{k^{\prime}} \delta_{l^{\prime}}^{j} \frac{\omega_{0}^{d}}{\left(\omega_{0}^{2}+\vec{\omega}^{2}\right)^{d}}-\frac{1}{2 d(d+1)} \partial_{k^{\prime}} \partial_{i} \frac{\delta_{l^{\prime}}^{j} \omega_{0}^{d}}{\left(\omega_{0}^{2}+\vec{\omega}^{2}\right)^{d-1}}\right. \\
& -\frac{1}{2 d(d+1)} \partial_{l^{\prime}} \partial_{j} \frac{\delta_{i}^{k^{\prime}} \omega_{0}^{d}}{\left(\omega_{0}^{2}+\vec{\omega}^{2}\right)^{d-1}}+\frac{1}{2(d-1) d(d+1)} \partial_{k^{\prime}} \partial_{l^{\prime}} \frac{\delta_{i}^{j} \omega_{0}^{d}}{\left(\omega_{0}^{2}+\vec{\omega}^{2}\right)^{d-1}} \\
& \left.+\frac{1}{4(d-2)(d-1) d(d+1)} \partial_{i} \partial_{j} \partial_{k^{\prime}} \partial_{l^{\prime}} \frac{\omega_{0}^{d}}{\left(\omega_{0}^{2}+\vec{\omega}^{2}\right)^{d-2}}\right) \\
& \mathcal{E}_{i j, i^{\prime} j^{\prime}} \mathcal{E}_{k l, k^{\prime} l^{\prime}}\left(J_{i^{\prime}}^{k^{\prime} J_{l^{\prime}}^{j^{\prime}}}\right)(\omega) \frac{\omega_{0}^{d}}{\left(\omega_{0}^{2}+\vec{\omega}^{2}\right)^{d}}=\mathcal{E}_{i j, i^{\prime} j^{\prime}} \mathcal{E}_{k l, k^{\prime} l^{\prime}} \\
& \times\left(\frac{d-1}{d+1} \delta_{i^{\prime}}^{k^{\prime}} \delta_{l^{\prime}}^{j^{\prime}} \frac{\omega_{0}^{d}}{\left(\omega_{0}^{2}+\vec{\omega}^{2}\right)^{d}}-\frac{1}{d(d+1)} \partial_{k^{\prime}} \partial_{j^{\prime}} \frac{\delta_{l^{\prime}}^{i^{\prime}} \omega_{0}^{d}}{\left(\omega_{0}^{2}+\vec{\omega}^{2}\right)^{d-1}}\right. \\
& \left.+\frac{1}{4(d-2)(d-1) d(d+1)} \partial_{i^{\prime}} \partial_{j^{\prime}} \partial_{k^{\prime}} \partial_{l^{\prime}} \frac{\omega_{0}^{d}}{\left(\omega_{0}^{2}+\vec{\omega}^{2}\right)^{d-2}}\right) \\
& \mathcal{E}_{k l, k^{\prime} l^{\prime}}\left(J_{i}^{k^{\prime}} J_{l^{\prime}}^{0}\right)(\omega) \frac{\omega_{0}^{d}}{\left(\omega_{0}^{2}+\vec{\omega}^{2}\right)^{d}}=\frac{d-1}{d(d+1)} \mathcal{E}_{k l, i m} \partial_{m} \frac{\omega_{0}^{d+1}}{\left(\omega_{0}^{2}+\vec{\omega}^{2}\right)^{d}}-\frac{1}{2(d-1) d(d+1)} \mathcal{E}_{k l, k^{\prime} l^{\prime}} \partial_{k^{\prime}} \partial_{l^{\prime}} \partial_{i} \frac{\omega_{0}^{d+1}}{\left(\omega_{0}^{2}+\vec{\omega}^{2}\right)^{d-1}},
\end{aligned}
$$

and

$$
\mathcal{E}_{i j, i^{\prime} j^{\prime}}\left(J_{i^{\prime}}^{0} J_{0}^{j^{\prime}}\right)(\omega) \frac{\omega_{0}^{d}}{\left(\omega_{0}^{2}+\vec{\omega}^{2}\right)^{d}}=\frac{1}{d(d+1)} \mathcal{E}_{i j, i^{\prime} j^{\prime}} \partial_{i^{\prime}} \partial_{j^{\prime}} \frac{\omega_{0}^{d+2}}{\left(\omega_{0}^{2}+\vec{\omega}^{2}\right)^{d}}
$$

One can rewrite every $I^{k}$ as derivatives with respect to the external variable $t_{i}=z_{i}^{\prime}-y_{i}^{\prime}$ of the standard integrals [7]:

$$
\begin{aligned}
I_{b, c}^{a}= & \int d^{d+1} \omega \frac{\omega_{0}^{a}}{\left[\omega_{0}^{2}+\vec{\omega}^{2}\right]^{b}\left[\omega_{0}^{2}+(\vec{\omega}-\vec{t})^{2}\right]^{c}}=\frac{\pi^{d / 2}}{2} \frac{\Gamma(a / 2+1 / 2) \Gamma(b+c-d / 2-a / 2-1 / 2)}{\Gamma(b) \Gamma(c)} \\
& \times \frac{\Gamma(1 / 2+a / 2+d / 2-b) \Gamma(1 / 2+a / 2+d / 2-c)}{\Gamma(1+a+d-b-c)}|\vec{x}-\vec{y}|^{1+a+d-2 b-2 c} .
\end{aligned}
$$

After straightforward calculations one obtains that all integrals $I^{k}$ result in the same form

$$
\begin{aligned}
I^{k}= & \frac{\pi^{d / 2}}{2} \frac{\Gamma(d / 2)[\Gamma(d / 2+1)]^{2}}{(d+1)^{2} \Gamma(d-1) \Gamma(d+2)} \frac{1}{|t|^{d}}\left\{+a_{1}^{(k)} \mathcal{E}_{i j, a b} \mathcal{E}_{k l, a c} \mathcal{E}_{m n, b c}+a_{2}^{(k)} \mathcal{E}_{i j, a b} \mathcal{E}_{k l, a c} \mathcal{E}_{m n, b d} \frac{t_{c} t_{d}}{t^{2}}\right. \\
& +a_{3}^{(k)}\left[\mathcal{E}_{k l, a b} \mathcal{E}_{m n, b c} \mathcal{E}_{m n, a d}+(k l) \rightarrow(m n)\right] \frac{t_{c} t_{d}}{t^{2}}+a_{4}^{(k)}\left[\mathcal{E}_{k l, i j}\left(\frac{t_{m} t_{n}}{t^{2}}-\frac{1}{d} \delta_{m n}\right)+(k l) \rightarrow(m n)\right]+a_{5}^{(k)} \mathcal{E}_{k l, m n}\left(\frac{t_{i} t_{j}}{t^{2}}-\frac{1}{d} \delta_{i j}\right) \\
& +a_{6}^{(k)}\left[\mathcal{E}_{k l, a b} \mathcal{E}_{i j, a c}\left(\frac{t_{m} t_{n}}{t^{2}}-\frac{1}{d} \delta_{m n}\right)+(k l) \rightarrow(m n)\right] \frac{t_{b} t_{c}}{t^{2}}+a_{7}^{(k)} \mathcal{E}_{k l, a b} \mathcal{E}_{m n, a c}\left(\frac{t_{i} t_{j}}{t^{2}}-\frac{1}{d} \delta_{i j}\right) \frac{t_{b} t_{c}}{t^{2}} \\
& \left.+a_{8}^{(k)}\left(\frac{t_{i} t_{j}}{t^{2}}-\frac{1}{d} \delta_{i j}\right)\left(\frac{t_{k} t_{l}}{t^{2}}-\frac{1}{d} \delta_{k l}\right)\left(\frac{t_{m} t_{n}}{t^{2}}-\frac{1}{d} \delta_{m n}\right)\right\}
\end{aligned}
$$


but with different coefficients $a_{i}^{(k)}$, where $i=1, \ldots, 8$ and $k=1,2,3$. be

For the first integral $I^{1}$ the coefficients $a_{i}^{(k)}$ are found to

$$
\begin{aligned}
& a_{1}^{(1)}=\frac{4(d-1)(d+1)}{d}+\frac{(d-1)(8 d+12)}{d^{2}}+\frac{8 d+4}{d^{2}} \\
& +\frac{12(d+2)+8(d-2)(d+3)}{d^{2}(d-1)(d+3)}, \\
& a_{2}^{(1)}=-\frac{2(d-1)(2 d+3)}{d}-\frac{8 d+4}{d} \\
& -\frac{12(d+2)+8(d-2)(d+3)}{d(d-1)(d+3)}, \\
& a_{3}^{(1)}=-6-\frac{2(d-1)(d+1)}{d} \\
& -\frac{12(d+2)+8(d-2)(d+3)}{d(d-1)(d+3)}, \\
& a_{4}^{(1)}=-\frac{(d+3)(d-3)+3(d+2)}{d(d-1)(d+3)}, \\
& a_{5}^{(1)}=-\frac{d-1}{d}-\frac{3(d+2)+2(d-2)(d+3)}{d(d-1)(d+3)} \text {, } \\
& a_{6}^{(1)}=\frac{(d+2)(2 d+1)}{d} \\
& +\frac{(d+2)[6(d+2)+4(d-2)(d+3)]}{d(d-1)(d+3)}, \\
& a_{7}^{(1)}=\frac{(d-1)(d+2)}{d} \\
& +\frac{(d+2)[6(d+2)+4(d-2)(d+3)]}{d(d-1)(d+3)},
\end{aligned}
$$

$$
a_{8}^{(1)}=-\frac{(d+2)(d+4)[3(d+2)+2(d-2)(d+3)]}{2 d(d-1)(d+3)} .
$$

For the second integral $I^{2}$ the coefficients $a_{1}^{(2)}, \ldots, a_{8}^{(2)}$ are as follows:

$$
\begin{gathered}
a_{1}^{(2)}=\frac{2(d-1)}{d}+\frac{4}{d}+\frac{4(d+2)}{d(d-1)(d+3)}, \\
a_{2}^{(2)}=-2(d-1)-4-\frac{4(d+2)}{(d-1)(d+3)}, \\
a_{3}^{(2)}=-2-\frac{4(d+2)}{(d-1)(d+3)}, \quad a_{4}^{(2)}=-1-\frac{(d+2)}{(d-1)(d+3)}, \\
a_{5}^{(2)}=-\frac{(d+2)}{(d-1)(d+3)}, \quad a_{6}^{(2)}=d+2+\frac{2(d+2)^{2}}{(d-1)(d+3)}, \\
a_{7}^{(2)}=\frac{2(d+2)^{2}}{(d-1)(d+3)}, \quad a_{8}^{(2)}=-\frac{(d+2)^{2}(d+4)}{2(d+3)(d-1)},
\end{gathered}
$$

while for the third one they are

$$
\begin{aligned}
& a_{1}^{(3)}=-\frac{4}{d}-\frac{4(d+4)}{d(d-1)(d+3)}, \quad a_{2}^{(3)}=4+\frac{4(d+4)}{(d-1)(d+3)}, \\
& a_{3}^{(3)}=2+\frac{4(d+4)}{(d-1)(d+3)}, \quad a_{4}^{(3)}=d+1+\frac{(d+4)}{(d-1)(d+3)}, \\
& a_{5}^{(3)}=\frac{(d+4)}{(d-1)(d+3)}, \quad a_{6}^{(3)}=-d-2-\frac{2(d+2)(d+4)}{(d-1)(d+3)}, \\
& a_{7}^{(3)}=-\frac{2(d+2)(d+4)}{(d-1)(d+3)}, \quad a_{8}^{(3)}=\frac{(d+2)(d+4)^{2}}{2(d-1)(d+3)} .
\end{aligned}
$$

The coefficients $a_{i}^{(k)}$ are obtained for the general dimension $d$. We then specify their value for $d=4$ in Table I.
[1] J. Maldacena, Adv. Theor. Math. Phys. 2, 231 (1998).

[2] G. G. Gubser, I. R. Klebanov, and A. M. Polyakov, Phys. Lett. B 428, 105 (1998).

[3] E. Witten, Adv. Theor. Math. Phys. 2, 253 (1998).

[4] I. Ya. Aref'eva and I. V. Volovich, "On large-N conformal field theories, field theories in anti-de Sitter space and singletons," hep-th/9803028.

[5] M. Henningson and K. Sfetsos, Phys. Lett. B 431, 63 (1998).

[6] W. Mück and K. S. Viswanathan, Phys. Rev. D 58, 041901 (1998).

[7] D. Freedman, Samir D. Mathur, A. Matusis, and L. Rastelli, "Correlation functions in the $\mathrm{CFT}_{d} / \mathrm{AdS}_{d+1}$ correspondence,', hep-th/9804058.
[8] H. Liu and A. A. Tseytlin, Nucl. Phys. B533, 88 (1998).

[9] G. Chalmers, H. Nastase, K. Schalm, and R. Siebelink, Nucl. Phys. B540, 247 (1990).

[10] W. Mück and K. S. Viswanathan, Phys. Rev. D 58, 106006 (1998).

[11] A. Ghezelbash, K. Kaviani, S. Parvizi, and A. Fatollahi, Phys. Lett. B 435, 291 (1998).

[12] S. Lee, S. Minwalla, M. Rangamani, and N. Seiberg, Adv. Theor. Math. Phys. 2, 697 (1998).

[13] G. Arutyunov and S. Frolov, Nucl. Phys. B544, 576 (1999).

[14] G. Arutyunov and S. Frolov, Phys. Lett. B 441, 173 (1998).

[15] S. Corley, Phys. Rev. D 59, 086003 (1999).

[16] A. Volovich, J. High Energy Phys. 09, 022 (1998). 
[17] W. S. l'Yi, "Generating functionals of correlation functions of $p$-form currents in the AdS/CFT correspondence,', hep-th/0909132.

[18] W. Mück and K. S. Viswanathan, "The graviton in the AdS/ CFT correspondence: Solution via the Dirichlet boundary value problem," hep-th/9810151.

[19] L. Chekhov, "AdS/CFT correspondence on torus,", hep-th/9811146.

[20] A. Koshelev and O. Rytchkov, Phys. Lett. B 450, 368 (1999).

[21] H. Liu and A. A. Tseytlin, Phys. Rev. D 59, 086002 (1999).

[22] D. Freedman, S. D. Mathur, A. Matusis, and L. Rastelli, "Comments on 4-point functions in the CFT/AdS correspondence," hep-th/9808006.

[23] G. Chalmers and K. Schalm, "The large $N_{c}$ limit of four-point functions in $N=4$ super Yang-Mills theory from Anti-de Sitter supergravity,', hep-th/9810051.

[24] E. D'Hoker and D. Freedman, Nucl. Phys. B544, 612 (1999).

[25] J. H. Brodie and M. Gutperle, Phys. Lett. B 445, 296 (1999).

[26] H. Liu, "Scattering in Anti-de Sitter space and operator prod- uct expansion,' Report No. IMPERIAL-TP-98-99/12, hep-th/9811152.

[27] E. D 'Hoker and D. Freedman, "General scalar exchange in $\operatorname{AdS}_{d+1}$,' Report Nos. UCLA/98/TEP/34, MIT-CTP-2795, hep-th/9811257.

[28] G. Arutyunov and S. Frolov, "Quadratic action for type IIB supergravity on $\mathrm{AdS}_{5} \times S^{5}$,', hep-th/9811106.

[29] J. Erdmenger and H. Osborn, Nucl. Phys. B483, 431 (1997).

[30] E. D'Hoker, D. Freedman, and W. Skiba, Phys. Rev. D 59, 045008 (1999)

[31] P. S. Howe, E. Sokatchev, and P. C. West, Phys. Lett. B 444, 341 (1998).

[32] G. W. Gibbons and S. W. Hawking, Phys. Rev. D 15, 2752 (1977).

[33] H. Osborn and A. Petkou, Ann. Phys. (N.Y.) 231, 311 (1994).

[34] A. Strominger, Phys. Lett. B 383, 44 (1996).

[35] E. Witten, Nucl. Phys. B463, 383 (1996).

[36] N. Seiberg, Nucl. Phys. B (Proc. Suppl.) 67, 158 (1998).

[37] P. Claus, R. Kallosh, and A. Van Proeyen, Nucl. Phys. B518, 117 (1998). 